\title{
Joint analysis of the ionospheric parameters and cosmic ray data during periods of magnetic storms 2015
}

\author{
Oksana Manrikova ${ }^{1}$, Yury Polozov ${ }^{1, *}$, and Timur Zalyaev ${ }^{1}$ \\ ${ }^{1}$ Institute of Cosmophysical Researches and Radio Wave Propagation, Mirnaya Str., 7, 684034, \\ Kamchatka Region, Elizovskiy District, Paratunka 684034, Russia
}

\begin{abstract}
Method of joint analysis of ionospheric parameters and cosmic ray data is proposed. The method allowsto allocate anomalous changes in data before strong magnetic storms. The developed method is based on the application of wavelet transform, neural networks and classical autoregressive models. The application of the method has shown its effectiveness and the possibility of using in the problems of estimating space weather and predicting strong magnetic storms. The research was supported by RSF Grant, project No 14-11-00194.
\end{abstract}

\section{Introduction}

The work is aimed at studying processes in the near-Earth space during periods of increased solar activity and magnetic storms. At present, many issues of energy transfer in the solar wind-magnetosphere-ionosphere system remain open, which determines the relevance of these studies. In the article we performed a joint analysis of ionospheric parameters and galactic cosmic ray data (GCR). It is known that the most significant changes in the parameters of GCR are caused by the ejections of the coronary mass and the following changes in the parameters of the interplanetary field and the solar wind [1,2]. GCR observations are used in a number of fundamental and applied studies related to monitoring and forecasting space weather $[2,3]$. The GCR variations have a complicated structure, their dynamics reflect the 11-year cycle and the 27-day solar rotation period [4] and there is a diurnal variation due to the asymmetry of the shape of the magnetosphere, which varies with time when the solar wind parameters change [5]. Traditional methods of data analysis are not sufficiently effective for their investigation [1,5-7]. The analysis of GCR in the work is based on the joint application of wavelet transform and neural network methods, which are currently gaining intensive development in this field [1,6-12]. The method of

* Corresponding author: up agent@mail.ru 
joint application of mathematical devices for studying complex time series was first proposed by the authors in works [7, 10, 12-14] The method allows to study in detail the data structure, to determine typical variations and to allocate anomalies. Its application allowed us to isolate the anomalous effects in the dynamics of GCR (increases that occur 820 hours before the onset of magnetic storms) $[7,10,11]$ arising on the eve of geomagnetic storms. These anomalies were first discovered by statistical analysis and described in [15]. The results of the analysis presented in this paper confirm the possibility of the appearance of the effect of an increase in GCR and show the effectiveness of the proposed technique for their detection..

A similar approach based on the combination of wavelet transform and neural networks was developed by the authors to study the dynamics of ionospheric parameters $[13,14,16]$ (the data of the critical frequency of the F2 layer of the ionosphere (foF2) and PES data were analyzed). During the periods of disturbances, anomalous changes are observed in the recorded ionospheric parameters, which indicate the occurrence of anomalous processes in the ionosphere. It is known that the strongest ionospheric disturbances occur during solar events and geomagnetic storms, the study of which has important scientific and applied significance [17-21]. In this paper, based on the joint analysis of the ionosphere parameters and GCR data on the eve of magnetic storms, anomalous increases in variations in the intensity of cosmic rays and the increase in the electron density of the ionosphere that arise during these periods are likely to be associated with the approaching events.

\section{Methods of data analysis}

\subsection{Modeling of the CR time series and isolation of abnormal changes.}

1 On the basis of the multi-scale wavelet transform [22, 23], a time series of GCR data was obtained in the form:

$f(t)=f_{a,(-6)}(t)+\sum_{j=-1}^{-6} f_{d, j}(t)$

where $f_{a,(-6)}(t)=\sum_{n} c_{-6, n} \phi_{-6, n}(t), f_{d, j}(t)=\sum_{n} d_{j, n} \Psi_{j, n}, \Psi_{j}=\left\{\Psi_{j, n}\right\}_{n \in Z}-$ waveletbasis, $\varphi_{j}=\left\{\varphi_{j, n}\right\}_{n \in Z}$, basis, generated by scaling function, $j$-scale.

2. Approximation of the characteristic component of the time series of GCR based on the neural network:

$$
\overline{\overline{c_{-m, n+1}}}=\phi_{k}^{3}\left(\sum_{i} \omega_{k i} \phi_{i}^{2}\left(\sum_{l} \omega_{i l} \phi_{l}^{1}\left(\sum_{\mathrm{z}=0}^{\gamma} \omega_{l n} c_{-m, n-z}\right)\right)\right)
$$

where $\omega_{k i}, \omega_{i l}, \omega_{l n}-$ weights of network,$\phi_{l}^{1}=\phi_{i}^{2}=\frac{2}{1+e^{-2 z}}-1 ; \phi_{k}^{3}=a * z+b ; \gamma-$ dimension of the input vector of the network.

Estimates of the adequacy of this model are given in [24]. The architecture of the constructed neural network is shown in Fig.1. 


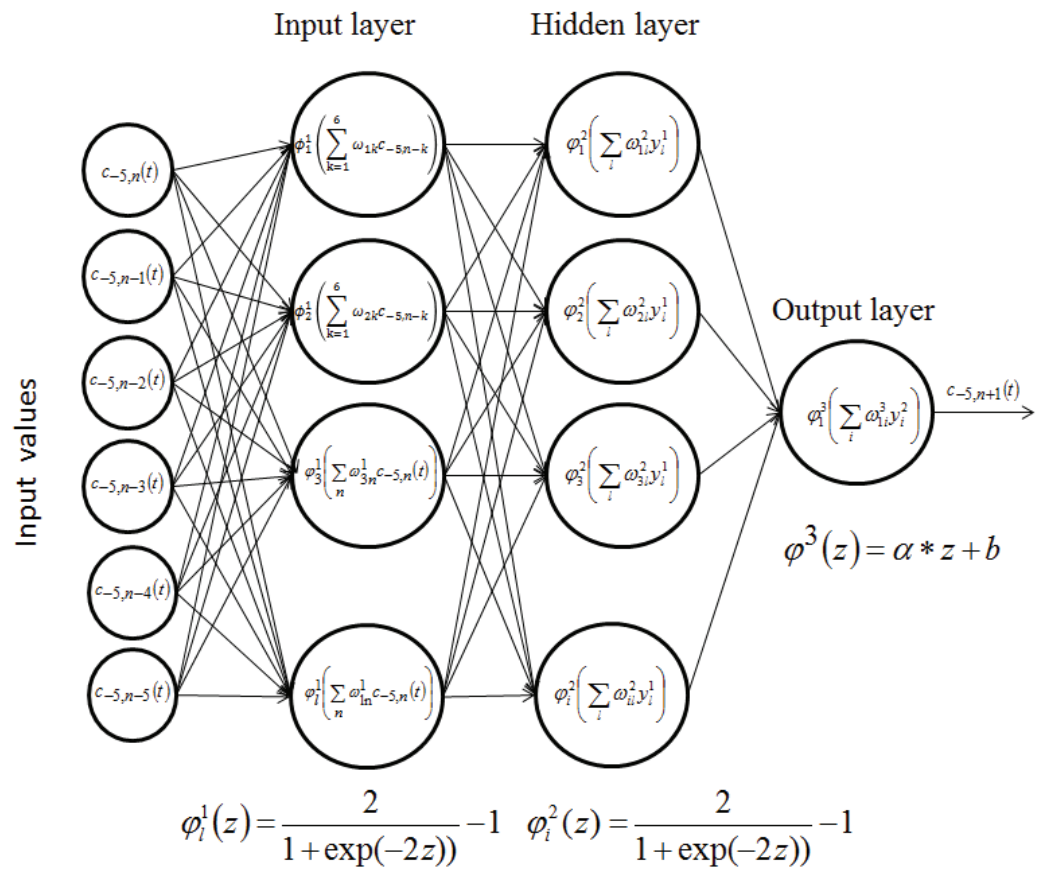

Fig.1. The architecture of a neural network that approximates the time series of a typical GCR component.

Performing the operation (2) allows to reproduce typical variations of the GCR (approximates the typical level of FCR variations). In the period of anomalous changes in the time series of the GCR, the absolute values of the errors of the trained NN increase, so the operation of their isolation can be based, for example, on checking the following condition:

$$
|e(t)|>T_{s}
$$

where $T_{\mathrm{s}}$ - threshold value for anomaly. As the threshold $T_{\mathrm{s}}$ the threshold $T_{\mathrm{s}}=2.5 \sigma_{\text {calm }}$, was used in the work, where $\sigma_{\text {calm }}$ - is the root-mean-square error of the $\mathrm{NN}$ for the periods used in training..

\subsection{Allocation of short-term anomalies in the CR variations.}

Anomalies in recorded GCR variations may contain large-scale (trend) changes occurring during periods of prolonged Forbush effects, and may also contain short-term anomalous features characteristic of local GCR enhancements and depressions. The above neural network model (2) approximates the characteristic variations of GCR and on its basis largescale GCR anomalies can be distinguished. To identify short-term anomalies, the authors developed computational solutions based on the continuous wave-transformation, first proposed in [25]:

1. Continuous wavelet transform of the data [22]:

$$
\left(W_{\Psi} f_{b, s}\right):=|s|^{-\frac{1}{2}} \int_{-\infty}^{+\infty} f(t) \Psi\left(\frac{t-b}{s}\right) d t
$$


where $f \in L^{2}(R), s, b \in R, s \neq 0, \Psi$ - basis wavelet, parameter $s$ characterizes the scale, $b$ - time moment.

2. Application of threshold function:

$$
\mathrm{P}_{T_{s}^{l}}^{\mathrm{CR}}\left(W_{\Psi} f_{b, s}\right)=\left\{\begin{array}{c}
W_{\Psi} f_{b, s}, \text { if }\left(W_{\Psi} f_{b, s}-W_{\Psi} f_{b, s}^{\text {med }, l}\right) \geq T_{s}^{l} \\
0, \text { if }\left|W_{\Psi} f_{b, s}-W_{\Psi} f_{b, s}^{\text {med,l }}\right|<T_{s}^{l} \\
-W_{\Psi} f_{b, s}, \text { if }\left(W_{\Psi} f_{b, s}-W_{\Psi} f_{b, s}^{\text {med,l }}\right)<-T_{s}^{l}
\end{array}\right.
$$

where $W_{\Psi} f_{b, s}^{\text {med,l}}$-median value, calculated in a sliding time window of length $l . T_{s}^{l}=U *$ $\sigma_{s}^{l}-$ threshold function, where $\sigma_{s}^{l}=\sqrt{\left(\frac{1}{l}-1 \sum_{k=1}^{l}\left(W_{\Psi} f_{b, s}-\overline{W_{\Psi} f_{b, s}}\right)^{2}\right.}-$ is the standard deviation calculated in a sliding time window of length $l, \overline{W_{\Psi} f_{b, s}}-$ median value, $U$ is a threshold coefficient.

The choice of the threshold coefficient $U$ is based on the assessment of a posteriori risk, the results of experiments with real data and the results of statistical modeling were used in the estimates. The model data correspond to the neutron monitors data (the data of the Apatity stations, Cape Schmidt and Magadan were used), they contain a recurrence component of the variations and different-scale local features, and white noise. The results of estimating the efficiency of the algorithm for different values of the $U$ coefficient on the model data are given in Table 1 . In accordance with the estimates, $U=2.5$ was used in the work.

The choice of the analyzing wavelet $\Psi$ was based on the following criteria: the number of zero moments, the smoothness of the wavelet and the size of the carrier. Taking into account the chosen criteria, the basic wavelet Coiflet of order 1 was used (has the least possible length of the carrier and ensures the detection of the feature).

Table 1. Estimation of the efficiency of the algorithm for different values of the threshold coefficient .

\begin{tabular}{|l|l|l|l|l|}
\hline $\begin{array}{l}\text { Analyzing } \\
\text { wavelet }\end{array}$ & $\begin{array}{l}\text { Percentage of } \\
\text { isolated positive } \\
\text { anomalies }\end{array}$ & $\begin{array}{l}\text { Percentage of } \\
\text { isolated } \\
\text { negative } \\
\text { anomalies }\end{array}$ & $\begin{array}{l}\text { Percentage of } \\
\text { false positives }\end{array}$ & $U$ \\
\hline coif1 & $89 \%$ & $97 \%$ & $27 \%$ & 2,0 \\
\hline coif1 & $88 \%$ & $94 \%$ & $24 \%$ & 2,1 \\
\hline coif1 & $86 \% \%$ & $91 \%$ & $21 \%$ & 2,2 \\
\hline coif1 & $85 \%$ & $89 \%$ & $19 \%$ & 2,3 \\
\hline coif1 & $84 \%$ & $86 \%$ & $17 \%$ & 2,4 \\
\hline coif1 & $83 \%$ & $85 \%$ & $15 \%$ & 2,5 \\
\hline
\end{tabular}

The length of the sliding time window $l=1440$ counts, which corresponds to one day (it is determined taking into account the diurnial variations of the GCR).

The use of operation (5) allows one to fix the periods of anomalous increases and abnormal GCR decreases.

3. To estimate the intensity of the anomaly at time $t=b$, we used the value :

$Y_{b}=\sum_{s} \mathrm{P}_{T_{s}^{l}}^{\mathrm{CR}}\left(W_{\Psi} f_{b, s}\right)$, 
which in the case of a local increase in GCR is positive, and in the case of a local decrease in GCR, is negative.

\subsection{Detailed analysis of ionospheric parameters.}

1. The initial time series of ionospheric parameters is represented in the form of wavelet coefficients obtained on the basis of a continuous wavelet decomposition [23] (see representation 4).

2. On the basis of the computational solutions proposed in [18], we determine the values of wavelet coefficients that exceed a given threshold:

$$
P_{Q_{s}}\left(W_{\Psi} f_{b, s}\right)=\left\{\begin{array}{l}
W_{\Psi} f_{b, s}, \text { если }\left|W_{\Psi} f_{b, s}-W_{\Psi} f_{b, s}^{\text {med }}\right| \geq Q_{s} \\
0, \text { если }\left|W_{\Psi} f_{b, s}-W_{\Psi} f_{b, s}^{\text {med }}\right|<Q_{s}
\end{array}\right.
$$

Where threshold $Q_{s}=\theta * S t_{s}$ - determines the presence of an anomaly on the scale $s$ near the point $\xi$ contained in a carrier $\Psi_{b, s}, \theta-$ threshold coefficient, $S t_{s}=\sqrt{\frac{1}{\Phi-1} \sum_{k=1}^{\Phi}\left(W_{\Psi} f_{b, s}-\overline{W_{\Psi} f_{b, s}}\right)^{2}}, \overline{W_{\Psi} f_{b, s}}$ и $W_{\Psi} f_{b, s}^{\text {med }}$ - mean and median values, defined in a sliding time window of length $\Phi$.

In work to select the threshold $Q_{s}$, the criterion of the lowest error rate was used (the a posteriori risk was assessed and minimized). When assessing a posteriori risk, the geomagnetic activity index $\mathrm{K}$ and the level of solar activity were used to determine the state of the ionosphere. The parameter values $\theta$ for the area Kamchatka were determined: $2.5 \leq \theta \leq 3.5$ - for analyzing data during periods of high solar activity (parameter $\left.f_{10.7}>100\right) ; 1.5 \leq \theta \leq 2.5$ - for analyzing data during periods of low solar activity (parameter $f_{10.7} \leq 100$ ).

3. The moments of exceeding the values of the wavelet coefficients show anomalous changes in the ionospheric parameters. An estimate of the intensity of the anomaly at time $t=b$ was fulfilled on the basis of the following formula:

$$
I_{b}=\sum_{s} \frac{\left|P_{Q_{s}}\left(W_{\Psi} f_{b, s}\right)\right|}{\left\|W_{\Psi} f_{b, s}\right\|_{2}},
$$

where norm $\left\|W_{\Psi} f_{b, s}\right\|_{2}=\sqrt{\sum_{N_{s}}\left(P_{Q_{s}}\left(W_{\Psi} f_{b, s}\right)\right)^{2}}, N_{s}$ - length of series on scale $s$.

\section{Results of data analysis}

In processing, we used minute data from neutron monitors of Kingston stations (Australia) and ionospheric data of Paratunka station (Russia). To assess the state of the geomagnetic field, the Dst component was used.

Fig. 2 shows the results of data analysis for the period January 20-23, 2015 during the magnetic storm. According to space weather data [http://ipg.geospace.ru/] on January 21, due to the accelerated flow from the coronal hole, the speed of the solar wind increased to $550 \mathrm{~km} / \mathrm{sec}$, the southern Bz component of the IMF dropped to $-15 \mathrm{nT}$, on January 22 the speed The solar wind remained within $550-400 \mathrm{~km} / \mathrm{s}, \mathrm{Bz}=-10 \mathrm{nT}$. Analysis of galactic cosmic ray data shows the appearance of a positive anomaly about 20 hours before the 
onset of the magnetic storm (shown in Fig. 2 in red). A few hours before the storm began, the level of GCR dropped (shown in Fig. 2 e in blue). The error of the neural network during the period of lowering level of GCR increased five-fold, and remained high for two days, which characterizes the occurrence of a deep and prolonged Forbush-lowering. During the period of lowering the level of GCR in the ionospheric parameters, a positive anomaly is observed (an abnormal increase in the electron concentration, shown in red in Fig. 2). During the main phase of the storm, the electron concentration remained elevated, the maximum anomaly reached at 05.00 UT on 22 January. Based on the results of processing events in the period 2010-2016. [26] such behavior of the ionosphere is not typical in the analyzed areas and can be observed during periods of moderate magnetic storms.

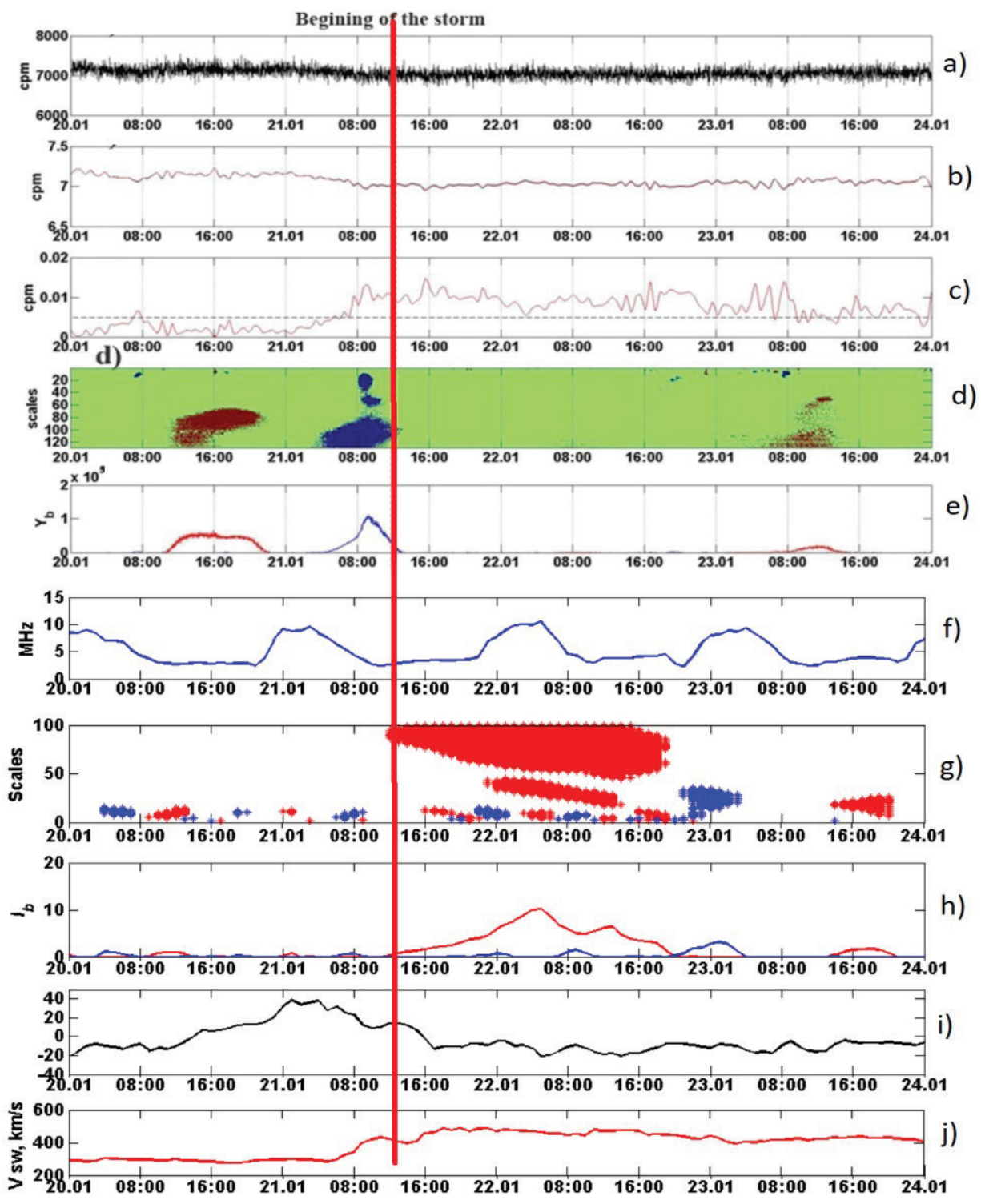

Fig. 2. Results of data processing for the period January 20-23, 2015 a) Data of the neutron monitor station Kingston; B) the quiet component of the neutron monitor data and its model, c) the neural 
network error; D) isolated positive (red) and negative (blue) anomalies; E) intensity of positive (red) and negative (blue) anomalies; F) - ionospheric data; G) - isolated ionospheric anomalies; $\mathrm{H}$ ) - the intensity of ionospheric anomalies; i) - Dst index; j) - solar wind speed..

Fig. 3 shows the results of data analysis for the period February 15-18, 2015. During this period, February 17, 2015, there was a magnetic storm. According to space weather [http://ipg.geospace.ru/] the effect of the solar wind velocity from the coronary hole in the evening time UT on February 16 led to a decrease in the Dst index to $-25 \mathrm{nT}$. Approximately at 6:00 UT on February 17, the speed of the solar wind increased to 380 $\mathrm{km} / \mathrm{s}$ and continued to grow until the evening of UT on February 17. During this period, the Dst-index continued to decline to $-50 \mathrm{nT}$. The results of the analysis of ionospheric parameters show the occurrence of a prolonged positive anomaly that arose on the eve of the storm (in Fig. 1 it is shown in red). Maximum values of the intensity of the ionospheric anomaly reached at 04.00 UT on February 17, 2015. At the end of the day on February 18, the electron concentration of the ionosphere decreased and a negative anomaly arose (in Figure 1 it is shown in blue). Analysis of galactic cosmic ray data shows that during the period of an anomalous increase in the electron concentration in the ionosphere, an appreciable increase in their intensity was observed (arose 20 hours before the beginning of the magnetic storm, in Fig. 1 it is shown in red). At the time of the onset of the magnetic storm, a negative anomaly is observed in the GCR data, which is probably related to the occurrence of a short Forbush decrease of a small amplitude. As a result of the processing of events in the period 2010-2016, [26] a similar behavior of the ionosphere is typical during periods of increased solar activity and strong magnetic storms. 


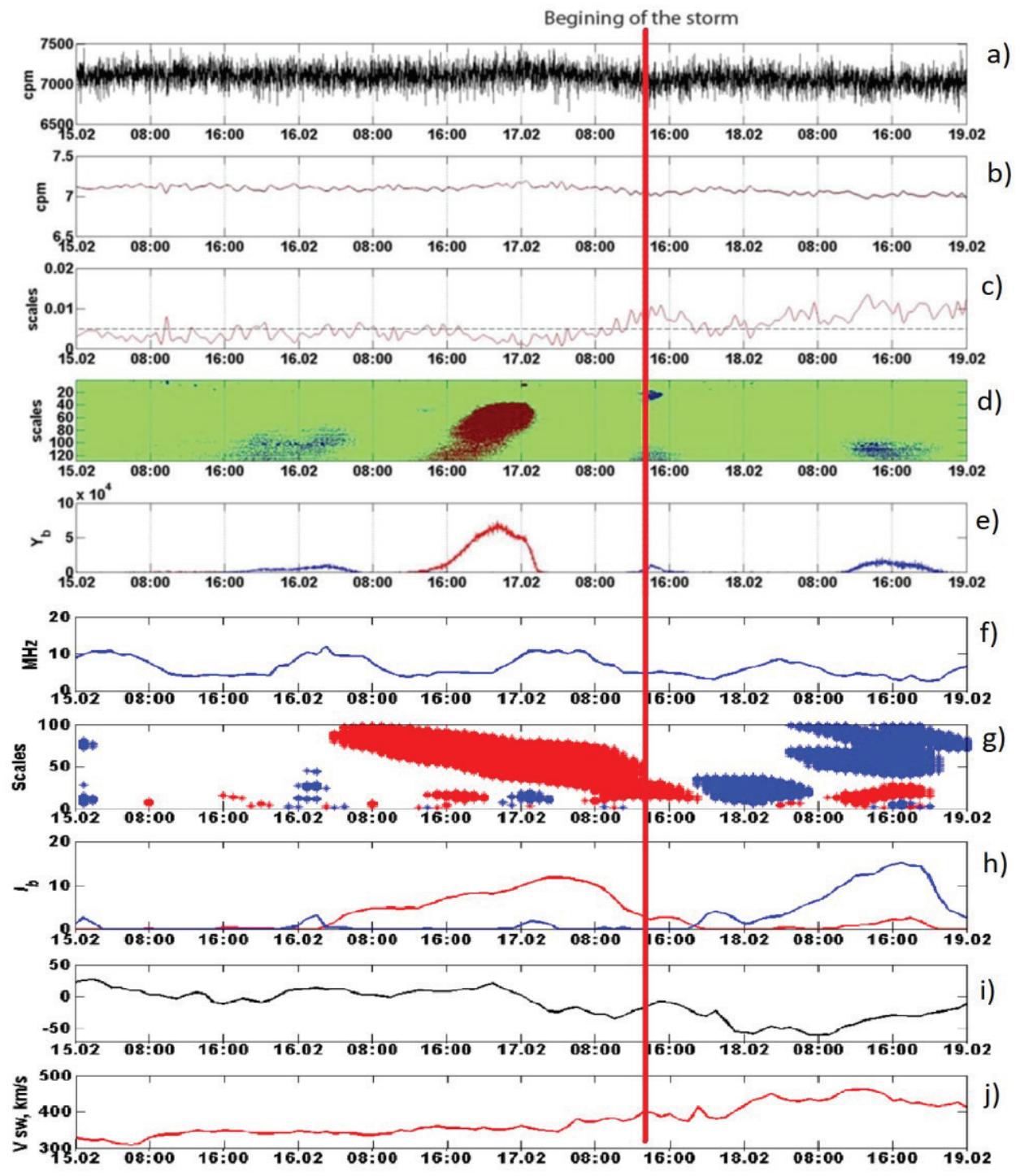

Fig. 3. Results of data processing for the period February 15-18, 2015a) data of the neutron monitor station Kingston; B) the quiet component of the neutron monitor data and its model, c) the neural network error; D) isolated positive (red) and negative (blue) anomalies; E) intensity of positive (red) and negative (blue) anomalies; F) - ionospheric data; G) - isolated ionospheric anomalies; H) - the intensity of ionospheric anomalies; i) - Dst index; j) - solar wind speed.

\section{Conclusions}

In the work, the fact of a possible anomalous increase in the intensity of GCR a few hours before the onset of strong magnetic storms was experimentally confirmed. In this case, these effects can be used as precursors of magnetic storms and be an additional factor in the tasks of space weather forecast. At the time of arrival of the shock wave, cosmic ray intensity decreases, typical to the Forbush decreases. An analysis of the ionospheric parameters showed that during periods of increasing galactic cosmic ray intensity in the 
analyzed regions, anomalous increases in the electron concentration in the ionosphere are typical. The results of [26 - 29] indicate repeated observations of this anomalous effect in the ionosphere on the eve of magnetic storms, but questions related to its nature and mechanisms remain open for the time being. The authors of the paper hold the opinion presented in [27] that such ionospheric anomalies are associated with a certain channel of energy penetration from interplanetary space and the magnetosphere. The study of the reaction of the ionosphere to anomalous changes in the intensity of galactic cosmic rays requires additional studies (using more representative statistics), and in the case of confirmation of the isolated effect, such pre-storm anomalies can serve as a signal for the forthcoming magnetic storm, which is of great practical importance.

The research is supported by a grant from the Russian Science Foundation (project No. 14-11-00194). The authors thank the institutions supporting the data recording stations that were used in the study, and also express their gratitude to the staff of the Institute of Terrestrial Magnetism, Ionosphere and Radio Wave Propagation RAS for the environment and database software that provide prompt information about the parameters of the state of outer space.

\section{References}

1. A. Vecchio, M. Laurenza, M. Storini, V. Carbone, Adv Astronomy, (2012)

2. L. M. Zelenyy, I. S. Veselovskiy, T. K. Breus, Plazmennaya geliogeofizika [Plasma geliogeofizika]. (Fizmatlit, Moscow, 2008)

3. I. N. Toptygin, Kosmicheskie luchi $\mathrm{v}$ mezhplanetnykh magnitnykh polyakh [Cosmicraysin the interplanetary magnetic field]. (Nauka, Moscow, 1983)

4. M. I. Tyasto, O. A. Danilova, V. M. Dvornikov, V. E. Sdobnov, Izvestiya RAN, ser. fizicheskaya [Izvestiya RAN, physical ser.], 73, (2008)

5. J. Kóta, A. Somogyi, Acta Physica Acad Sci Hung, 27, (1969)

6. P. Paschalis, C. Sarlanis, H. Mavromichalaki, Sol Phys, 182, (2013)

7. O. V. Mandrikova, T. L. Zalyaev, Tsifrovaya obrabotka signalov [Digital signal processing], 1, (2015)

8. V. I. Kozlov, V. V. Markov, Geomagnetism and Aeronomy, 47 (2007)

9. V. I. Kozlov, V. V. Markov, Geomagnetism and Aeronomy, 47 (2007)

10. V. I. Kozlov, V. V. Markov, Geomagnetism and Aeronomy, 48 (2008)

11. O. V. Mandrikova, T. L. Zalyaev, Mashinnoe obuchenie i analiz dannykh [Machine Learning Data Anal.], 1, (2014)

12. O. V. Mandrikova, I. S. Solovev, T. L. Zalyaev, Earth Plan. Space, 66, (2014)

13. O.V. Mandrikova, Yu.A. Polozov, V.V. Bogdanov, E.A. Zhizhikina A Journal of Software Engineering and Applications, 5, (2012)

14. O. Mandrikova, Yu. Polozov, I. Solovev, N. Fetisova, E3S Web of Conferences, 11, (2016)

15. A. V. Belov, J. W. Bieber, E. A. Eroshenko, P. Evenson, R. Pyle, V. G. Yanke, Adv. Space Res, 31, (2003)

16. O.V. Mandrikova, N.V. Glushkova, I.V. Zhivet'ev Geomagnetism and Aeronomy, 54 (2014)

17. D.G. Baishev, A.V. Moiseyev, R.N. Boroyev, S.E. Kobyakova, A.E. Stepanov, O.V. Mandrikova, I.S. Solovev, S.Yu. Khomutov, Yu.A. Polozov, A. Yoshikawa, K. Yumoto, Sun and Geosphere, 10, (2015) 
18. O.V. Mandrikova, N.V. Fetisova, Y.A. Polozov, I.S. Solovev, M.S. Kupriyanov, Earth, Planets and Space, 67, (2015)

19. O.V. Mandrikova, N.V. Fetisova (Glushkova), R.T. Al-Kasasbeh, D.M. Klionskiy, V.V. Geppener, M.Y. Ilyash, Annals of Geophysics, 58, 2015

20. O.V. Mandrikova, Yu.A. Polozov, I.S. Solovev, N.V. Fetisova(Glushkova), T.L. Zalyaev, M.S. Kupriyanov, A.V. Dmitriev Pattern recognition and image analysis, 26, (2016)

21. M. Nakamura, T. Maruyama, Y. Shidama Journal of the National Institute of Information and Communications Technology, 56, (2009)

22. C.K. Chui, An introduction in wavelets (Academic Press, New York, 1992)

23. I. Daubechies, CBMS-NSF lecture notes nr. (SIAM, Philadelphia, 1992)

24. O.V. Mandrikova, T. L. Zalyaev, SCM 2017, 1, (2017)

25. T. L. Zalyaev, News of "LETI" [Izvestija «LETI»], (2015)

26. O.V. Mandrikova, N.V. Fetisova, Yu.A. Polozov, Proceedings of the 11th Intl School and Conference "Problems of Geocosmos", (2017)

27. A.D. Danilov, Advances in Space Research, 52, (2013)

28. D. Buresova, J. Laštovička, Adv. Space Res., 39, (2007)

29. L. Liu, W. Wan, M.-L. Zhang, B. Zhao, Ann. Geophys., 26, (2008) 